\title{
Carbono y su costo social en América Latina
}

\section{Carbon and its social cost in Latin America}

\section{Magda Gabriela Sánchez-Trujillo ${ }^{1}$, Jorge Martín Hernández Mendoza ${ }^{2}$}

\begin{abstract}
:
The purpose of this document is to provide a summary of estimates of the social cost of carbon (CSC) with a view toward public policies concerning climate change in Latin America.

Models CSC seeking to have an estimate of the social, economic and environmental cost of climate change resulting from emissions of greenhouse gases into the atmosphere caused by economic activities affecting ecosystems and general welfare of the population of the planet. It is useful to identify the CSC as a mechanism in the construction of public policies on climate change and sustainable development.
\end{abstract}

Keywords:

Social cost of carbon, climate change, public policies

\section{Resumen:}

El objetivo de este documento, es ofrecer un resumen de las estimaciones del Costo Social del Carbono (CSC) con una visión hacia políticas públicas referentes al cambio climático en América Latina.

Los modelos de CSC buscan disponer de una estimación del costo social, económico y ambiental que ocasiona el cambio climático derivado de emisiones de gases de efecto invernadero a la atmosfera ocasionado por actividades económicas que afectan los ecosistemas y el bienestar en general de la población del planeta. Resulta útil identificar el CSC como un mecanismo en la construcción de políticas públicas al cambio climático y el desarrollo sostenible

\section{Palabras Clave:}

Costo social de carbono, cambio climático, políticas públicas.

\section{Introducción}

Al cambio climático se asocian fundamentos que acorde con la Organización Meteorológica Mundial, son variaciones en frecuencia de eventos extremos como (sequías, inundaciones y tormentas). Los científicos obtienen medida de las condiciones atmosféricas por periodos de aproximadamente 30 años con la finalidad de establecer medidas promedio y monitorear la variabilidad climática.

El conjunto de actividades económicas emite a la atmosfera gases contaminantes (de efecto invernadero) sin costo económico los cuales ocasionan el cambio en el clima. Es así que Hanley (2001) considera que atender esta condición de cambio climático conlleva la aplicación de políticas públicas, que podrían regular la contaminación, como sería el impuesto al carbono.

Ante esta propuesta, se requiere identificar los costos económico - sociales del carbono que ocasionan las emisiones de gases efecto invernadero a través del cambio climático, de esta manera se busca establecer un impuesto lo más objetivo posible ante el daño que ocasiona, ya que aún con el pago no se estará eliminando

\footnotetext{
${ }^{1}$ Profesora de Tiempo Completo. Programa Académico de Administración. Escuela Superior Tepeji del Rio. Universidad Autónoma del Estado de Hidalgo. ORCID http://orcid.org/0000-0002-9093-1081

${ }^{2}$ Profesor de Tiempo Completo. Programa Académico de Ingeniería Industrial. Escuela Superior Tepeji del Rio. Universidad Autónoma del Estado de Hidalgo. ORCID https://orcid.org/0000-0002-9262-4712
} 
la contaminación. Actualmente en diversos países se aplican impuestos al carbono y los rangos varían desde 1 dólar por tonelada en países como México, Polonia y Ucrania, hasta 126 dólares en Suecia. Existen 20 esquema de impuesto al carbono a nivel nacional y dos a nivel regional en Canadá (Ecofys, 2017), en promedio el valor de estos impuestos es de 22.1 dólares por tonelada. Así, el objetivo de este documento es presentar un resumen de estudios realizados por la Comisión Económica para América Latina (CEPAL) con el grado de incertidumbre que ello conlleva, dado que se trabaja bajo valoraciones económicas de varios bienes y servicios, así como simulaciones de escenarios a futuro. Sin embargo, es un momento para señalar los daños y poder instrumentar medidas de contingencia.

\section{La Ciencia del Clima}

Las emisiones de Gases efecto invernadero (GEI) tienen relación con el ciclo de vida del carbono y sus efectos en el clima (Clarkson y Deyes, 2002), destacan las siguientes:

Incertidumbre sobre la medición del nivel actual y futuro de las emisiones de GEI

Fluctuación sobre el ciclo de carbono y la capacidad de los océanos para almacenar $\mathrm{CO} 2$

Alteraciones sobre la sensibilidad climática, esto es sensibilidad de respuesta del clima ante las concentraciones de GEI (Clarkson y Deyes, 2002) Inseguridad en la obtención de cálculos precisos sobre efectos físicos y biológicos del cambio climático extremo. Como se puede observar, la incertidumbre es alta y afectada por supuestos exógenos, por tanto los costos económicos derivan en distintos parámetros y estimaciones (figura1).

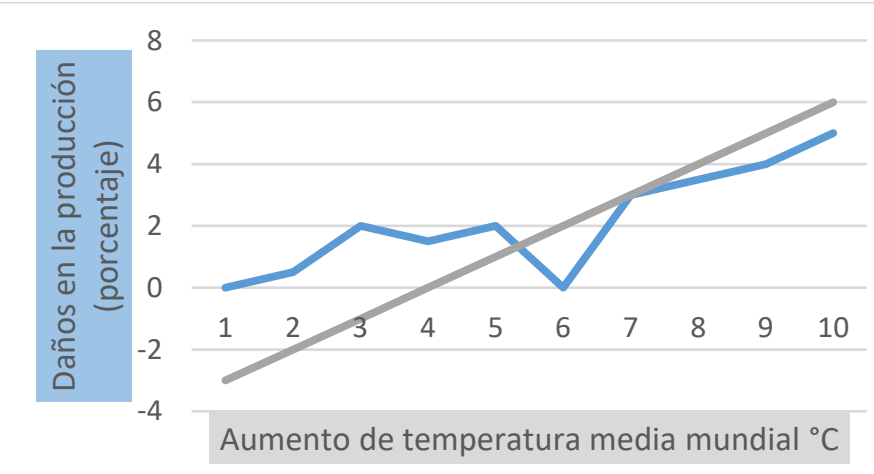

Figura 1. Estimaciones de impacto del cambio climático en la economía global. Nordhaus 2008.

Norhaus (2011) estimó un costo económico de $0.48 \%$ del PIB global actual con un aumento de $2.5{ }^{\circ} \mathrm{C}$ de temperatura y de $30 \%$ del PIB global actual con un aumento de $6^{\circ} \mathrm{C}$ de temperatura, cifras muy altas que ocasionarían daños catastróficos (figura 2).

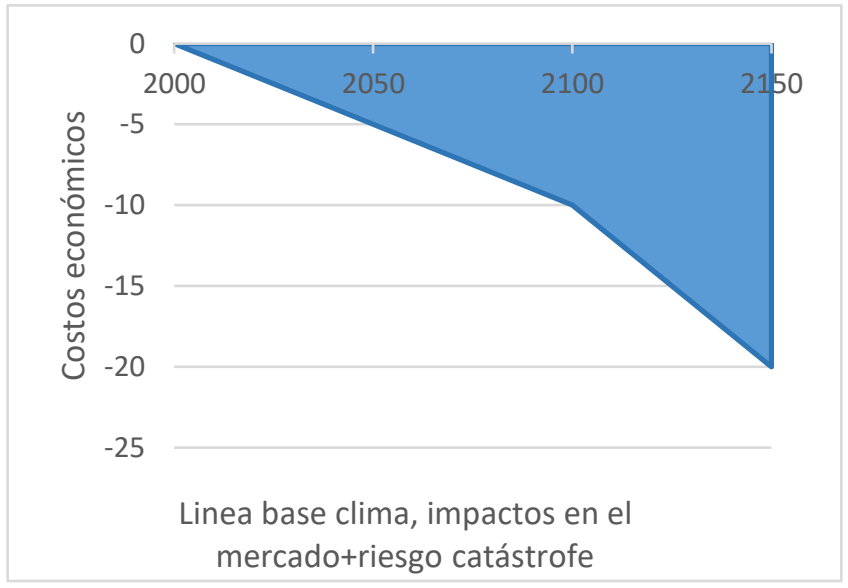

Figura 2. Impactos de cambio climático en el crecimiento y desarrollo. Stern 2017

Evolución de estimaciones al Costo Social del Carbono (CSC)

El Intergovernmental Panel of Climate Change (IPCC, 1996) por sus siglas en inglés realizo el estudio pionero sobre los rangos de impuestos por toneladas de carbono, los datos sugieren un rango entre 5 a 125 dólares por tonelada de carbono a precios del año 2000 y entre 7 y 154 para 2010, a precios de 1990. Tales cifras son valores ajustados que toman como evidencia recolectada en Estados Unidos.

En las últimas dos décadas, las estimaciones del CSC son derivadas de modelos integrados tales como DICE 
*(Dynamic Integrated Model of Climate-Economy model) y RICE (Regional Integrated Model of Climate-Economy model) son los más utilizados en economía del cambio climático.

El modelo PAGE (Policy Analysis of the Greenhouse Effect) (Hope, 2006), el modelo de Golosov et al (2014)

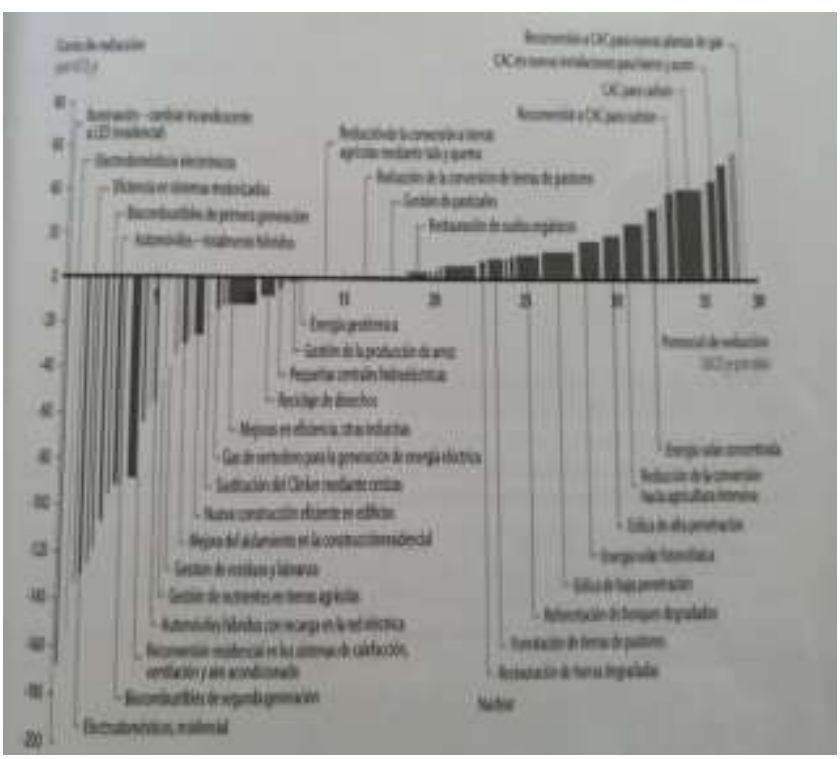

DSGE (Modelo dinámico estocástico de equilibrio general computable). Los diversos escenarios que permiten calcular los modelos conllevan diversidad de especificaciones y contextos, que por tanto alcanzan diversos resultados precisos.

Existen además estimaciones del CSC realizadas por gobiernos e instituciones públicas, como la Agencia de Protección Ambiental (EPA), los departamentos de agricultura, comercio, energía y transporte de la Casa Blanca, calculan una media de 26 dólares por tonelada de emisiones de CO2 para 2020 ( Greenstone et al., 2013) EL Banco Mundial establece un CSC de 20 dólares la tonelada de carbono con un rango entre 5 y 40 dólares para los proyectos de energía (Watkins et al, 2006) Los países Bajos (2001) propusieron un valor del CSC de 8.8 Euros la tonelada de carbono (Watkins et al, 2006). Por su parte, el Banco Europeo de Inversión propuso un valor entre 5 y 125 Euros por tonelada de carbono para

\footnotetext{
* Modelo DICE considera un modelo macroeconómico de crecimiento donde los combustibles fósiles son insumo de la producción que ocasiona emisiones de $\mathrm{CO}$. Aumento de
}

proyectos de energía, donde el rango bajo corresponde a daños de mercado y el rango alto daños a largo plazo (Watkins, 2006).

Las estimaciones para abatir los gases de efecto invernadero identifican costos específicos por fuentes de emisiones que conlleva a disminuirlas. Tales disminuciones, se denominan curvas marginales de abatimiento (CMA), representan la relación costoefectividad de las distintas opciones de reducción y la cantidad total de GEI disminuidas. Estas CMA reflejan los costos adicionales al reducir la última unidad de carbono y tienen pendiente ascendente, lo que significa que los costos marginales aumentan con el aumento del esfuerzo en reducción. De ahí que, las opciones para reducir costos podrían ser cambiar a energía limpia, mejorar la eficiencia energética, evitar deforestación, mejora de prácticas agrícolas y evitar quemar gas (figura 3).

El ancho de cada barra representa la eficacia de cada oportunidad para reducir las emisiones GEI en un año específico comparado con el desarrollo de actividades empresariales. El alto de cada barra representa el costo promedio de prevenir 1 tonelada de $\mathrm{CO} 2$ equivalente para el año 2030 a través de esa oportunidad. Se calcula el costo promedio ponderado a través de sub oportunidades, regiones y años. Además, el reporte de European Climate Change Programme estima costos de abatimiento para el protocolo de Kioto de 12 y 20 euros por tonelada de carbono en 2010 y 2020 respectivamente (Caballero, Galindo y Alatorre CEPAL 2019).

Figura 3. Curva costo de abatimiento de GEl a nivel Global 2030. MacKinsey \& Company (2019)

En este sentido, el uso de CMA es favorecido por los análisis de costo efectividad mientras que el uso del CSC considera el análisis de costo beneficio.

Otra forma indirecta de evaluar CSC es a través de la valoración contingente ${ }^{\dagger}$ de pagar por la aplicación de

emisiones, aumenta la temperatura y provoca efectos negativos en la economía.

${ }^{\dagger}$ Es un método directo de valoración que utiliza preferencia declaradas con base a mercado construido hipotéticamente 
políticas públicas que atiendan el desafío del cambio climático.

Se destaca que, las estimaciones del CSC derivan de un alto nivel de incertidumbre como consecuencia de la ciencia climática, como del uso de diversos métodos de análisis económico, social- demográfico y político*.

\section{Comentarios finales}

En el estudio del cambio climático, el costo social del carbono es un elemento fundamental de análisis que conlleva como parte de su solución al establecimiento de políticas públicas y una manera eficiente económicamente hablando, se basa en identificar los costos y beneficios potenciales aunado a la incertidumbre que envuelve los costos económicos, sociales y ambientales del cambio climático.

Las estimaciones como se ha señalado en el documento son heterogéneas y variadas. Los resultados del análisis de literatura indican una media de CSC de 25,83 dólares por tonelada de $\mathrm{CO} 2$, con una desviación de 0.84 . Además debe tomarse en cuenta que estas estimaciones excluyen normalmente, los efectos potenciales de eventos catastróficos extremos.

En suma, desde la perspectiva de la política pública, identificar el CSC en el largo plazo ofrece cierta certidumbre susceptible de convertirse en herramienta fundamental para la innovación en la producción, al sustituir bienes y servicios intensivos en carbono. De esta manera, el CSC resulta un mecanismo para coadyuvar a detener la deforestación, los subsidios a combustibles fósiles y mitigar sustancialmente el cambio climático (Madison, 1995).

\section{Referencias}

Caballero, K., Galindo, L.M., Alatorre, J. y Ferrer, J. (2019). Estudios del Cambio Climático en América Latina, División de Desarrollo Sostenible. CEPAL.

Clarkson, R. Y Deyes, K. (2002). Estimating the social cost of carbon emissions. Environment protection economics division, department of environment. Londres.

Greenstone, M. Kopits, E. Y Wolverton, A. (2013). Developing a social cost of carbon for US regulatory analysis: a methodology and interpretation. Review of environmental economics and policy 7(1), 23-46.

Hanley, N. (2001). Cost-benefit analysis and environmental policy making, Environment and planning $\mathrm{C}, 19,103-118$.

Hope, C. (2011). The social cost of CO2 from PAGE 09model. Economics ejournal, 39, 1-30.

Madison, D. (1995). A cost- benefit analysis of slowing climate change. Energy policy, 23 (4/5), 337-346.

McKinsey \& Company. (2019). Pathways to a LowCarbon Economy, Version 2 of the Global Greenhouse Gas Abatement Cost Curve.

Nordhaus, W. (2008). A question of Balance. Weighing the options on Global Warming Policies, Yale University Press, New Haven.

Nordhaus, W. (2011). Estimates of the social cost of carbon: background and results from the RICE2011Model. Cowles foundation for research in economics, Yale University, 1826, 1-48

Stern, N. (2017). The economics of Climate Change: The Stern Review. Cambridge

Watkins, P., Anthoff, D., Downing, T. y Tol, R.S.J. (2006). The social cost of carbon (SCC) reviewmethodological approaches for using SCC estimates in policy assessment, Final report to DEFRA.

World Bank. Ecofys- Vivid Economics. (2017). State and Trends of Carbon Pricing 2017. Washington, DC: World Bank. (c) World Bank. https://openknowledge.worldbank.org/handle/10986/2 8510 License: CC BY 3.0 IGO.

\footnotetext{
* Para mayor dato ver Clarkson y Deyes (2002) síntesis de estas incertidumbres.
}

(no experimental) para determinar la disposición de aceptar o pagar. 